\title{
Probing Axionlike Particles and the Axiverse with Superconducting Radio-Frequency Cavities
}

\author{
Zachary Bogorad, ${ }^{1, *}$ Anson Hook, ${ }^{2, \dagger}$ Yonatan Kahn, ${ }^{3,4, *}$ and Yotam Soreq ${ }^{5,6, \$}$ \\ ${ }^{1}$ Laboratory for Nuclear Science, Massachusetts Institute of Technology, Cambridge, Massachusetts 02139, USA \\ ${ }^{2}$ Maryland Center for Fundamental Physics, Department of Physics, University of Maryland, College Park, Maryland 20742, USA \\ ${ }^{3}$ Kavli Institute for Cosmological Physics, University of Chicago, Chicago, Illinois 60637, USA \\ ${ }^{4}$ University of Illinois Urbana-Champaign, Urbana, Illinois 61801, USA \\ ${ }^{5}$ Theoretical Physics Department, CERN, CH-1211 Geneva 23, Switzerland \\ ${ }^{6}$ Department of Physics, Technion, Haifa 32000, Israel
}

(Received 22 February 2019; published 9 July 2019)

\begin{abstract}
Axionlike particles (ALPs) with couplings to electromagnetism have long been postulated as extensions to the standard model. String theory predicts an "axiverse" of many light axions, some of which may make up the dark matter in the Universe and/or solve the strong CP problem. We propose a new experiment using superconducting radio-frequency (SRF) cavities which is sensitive to light ALPs independent of their contribution to the cosmic dark matter density. Off-shell ALPs will source cubic nonlinearities in Maxwell's equations, such that if a SRF cavity is pumped at frequencies $\omega_{1}$ and $\omega_{2}$, in the presence of ALPs there will be power in modes with frequencies $2 \omega_{1} \pm \omega_{2}$. Our setup is similar in spirit to light-shiningthrough-walls experiments, but because the pump field itself effectively converts the ALP back to photons inside a single cavity, our sensitivity scales differently with the strength of the external fields, allowing for superior reach as compared to experiments like OSQAR while utilizing current technology. Furthermore, a well-defined program of increasing sensitivity has a guaranteed physics result: the first observation of the Euler-Heisenberg term of low-energy QED at energies below the electron mass. We discuss how the ALP contribution may be separated from the QED contribution by a suitable choice of pump modes and cavity geometry, and conclude by describing the ultimate sensitivity of our proposed program of experiments to ALPs.
\end{abstract}

DOI: 10.1103/PhysRevLett.123.021801

Axions are well motivated new particles that have been proposed as a solution to the strong $C P$ problem [1-3] (see Refs. [4-6] for a review). Additionally, string theory, predicts a plethora of light $(\ll \mathrm{eV})$ particles [7], some of which may couple to electromagnetism in a manner very similar to the axion. These particles have been termed axionlike particles (ALP), and the (possibly) large number of ALPs, the "axiverse" [8]. One or more of these species may be excellent dark matter (DM) candidates [9-11], and/ or alleviate the hierarchy problem [12-16]. In light of this strong motivation, there has been much experimental effort devoted to the axion and its cousins [17,18].

There are several general approaches for finding ALPs, roughly analogous to the multipronged approach of direct detection, indirect detection, and collider production for WIMP DM. If the ALP makes up the DM of the Universe, it may be detected in the laboratory by converting ALPs to

Published by the American Physical Society under the terms of the Creative Commons Attribution 4.0 International license. Further distribution of this work must maintain attribution to the author(s) and the published article's title, journal citation, and DOI. Funded by SCOAP ${ }^{3}$. electromagnetic energy (see Refs. [19-21] for recent experimental results) or rotating the polarization of photons $[22,23]$, or in radio telescopes by searching for conversion [24-27] or decay $[28,29]$ to photons in astrophysical environments. Another approach which does not require the ALP to be DM is colloquially known as light shining through walls (LSW), where ALPs are both produced and detected in the laboratory. Such experiments are simultaneously sensitive to a wide range of ALP masses, and even multiple species of ALPs.

In this Letter, we propose a new experiment along the lines of an LSW experiment that utilizes light-by-light scattering mediated by off-shell ALPs, with production and detection taking place in a superconducting radio-frequency (SRF) cavity. An ALP $a$ is a pseudoscalar with Lagrangian

$$
\mathcal{L}_{a}=\frac{1}{2} \partial_{\mu} a \partial^{\mu} a-\frac{1}{2} m_{a}^{2} a^{2}-\frac{1}{4} g_{a \gamma \gamma} a F_{\mu \nu} \tilde{F}^{\mu \nu}
$$

For processes involving photons of energy $\omega$, an ALP with mass $m_{a} \gg \omega$ may be integrated out, giving an effective Lagrangian [30] 


$$
\mathcal{L}_{\mathrm{a}, \mathrm{eff}}=\frac{g_{a \gamma \gamma}^{2}}{32 m_{a}^{2}}\left(F_{\mu \nu} \tilde{F}^{\mu \nu}\right)^{2}
$$

In other words, an off-shell ALP will induce small nonlinearities in electromagnetism. Note that this effect is local, and does not require the ALP to propagate to another spacetime point to be converted back to photons. As we are also interested in very light ALPs, we will extend the analysis of Ref. [30] to the case where $m_{a} \ll \omega$. In this case the nonlinear effects are nonlocal, but we will show that detection proceeds similarly to the heavy ALP case.

Famously, loop contributions from virtual electrons will also induce such nonlinearities in pure quantum electrodynamics (QED), which are parametrized by the EulerHeisenberg (EH) Lagrangian [31,32]. To lowest order in $\alpha$ and $\omega / m_{e}$, this is

$$
\mathcal{L}_{\mathrm{EH}}=\frac{\alpha^{2}}{360 m_{e}^{4}}\left[4\left(F_{\mu \nu} F^{\mu \nu}\right)^{2}+7\left(F_{\mu \nu} \tilde{F}^{\mu \nu}\right)^{2}\right],
$$

valid for $\omega \ll m_{e}$. Light-by-light scattering with real photons has been observed at $\mathrm{GeV}$ energies $[33,34]$, but Eq. (3) has never been probed with real photons at $\omega<m_{e}$. Thus, an experiment that is designed to look for nonlinearities induced by ALPs would, if sensitive enough, also have the guaranteed physics result of discovering lightby-light scattering at low energies for the first time ever. Crucially, the effects of ALPs and the EH Lagrangian are not exactly degenerate, as the ALP Lagrangian only contains $F_{\mu \nu} \tilde{F}^{\mu \nu} \propto \mathbf{E} \cdot \mathbf{B}$, while the EH Lagrangian also contains $F_{\mu \nu} F^{\mu \nu} \propto \mathbf{E}^{2}-\mathbf{B}^{2}$. Thus, the two effects may be disentangled with a suitable choice of field configurations.

Comparing Eqs. (2) and (3), we expect the ALP contribution to four-photon processes to exceed the EH contribution when $[30,35]$

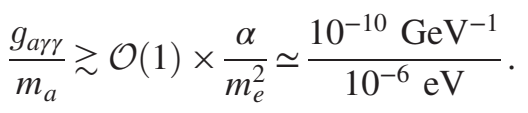

The best laboratory bounds on $g_{a \gamma y}$ are from the OSQAR [36] and PVLAS [37] experiments, which constrain $g_{\text {ary }}<3.5 \times 10^{-8} \mathrm{GeV}^{-1}$ for $m_{a} \lesssim 10^{-4} \mathrm{eV}$. Surpassing these bounds with a radio-frequency experiment $(\omega \sim$ $10^{-6} \mathrm{eV}$ ) would not require sensitivity to the $\mathrm{EH}$ Lagrangian, since the ALP term in the Lagrangian would be much larger. Under reasonable assumptions about solar physics, the bounds from the CAST experiment [38] constrain $g_{\text {ary }}<6.6 \times 10^{-11} \mathrm{GeV}^{-1}$ from thermal ALPs produced in the sun. (More stringent bounds can be obtained for $m_{a} \lesssim 10^{-10} \mathrm{eV}$ from the absence of photonALP oscillations in galactic magnetic fields [39-42].) Equation (4) shows that an experiment which surpasses these bounds would also probe the EH contribution.

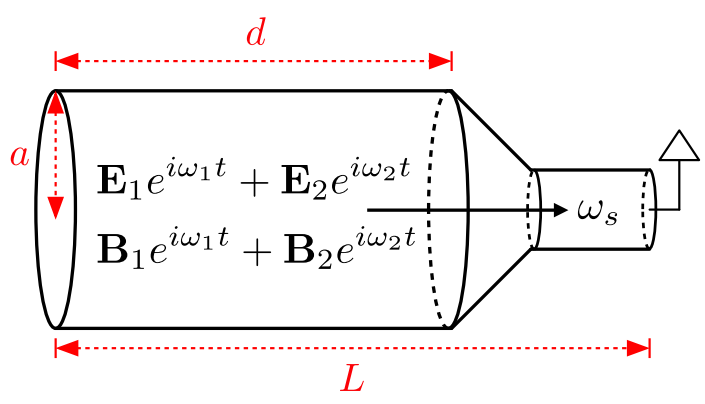

FIG. 1. Schematic of our proposed experiment, adapted from Ref. [44]. Note that $\omega_{s}$ extends through the bulk of the cavity, but the filtering geometry suppresses the pump fields in the detection region.

Detection strategy.-Taking these estimates as motivation, we extend the results of Refs. [43,44], a novel proposal for detecting the EH Lagrangian using SRF cavities, to include the contributions from the ALP Lagrangian (1). We will consider an SRF cavity pumped simultaneously at two frequencies $\omega_{1}$ and $\omega_{2}$, such that ALP- or EH-induced nonlinearities will give signal photons at $\omega_{s}=2 \omega_{1}-\omega_{2}$ (see Fig. 1).

While it was demonstrated in Ref. [35] that light-by-light scattering experiments will likely never be sensitive to the so-called QCD axion that solves the strong- $C P$ problem [1-3], renewed interest in the axiverse strongly motivates a reexamination of these results for general (non-DM, nonQCD) ALPs. Indeed, multiple ALPs will all contribute to nonlinearities in electromagnetism. Because our proposed experiment is sensitive to off-shell ALPs, our signal scales as $\mathcal{N}_{a}^{2} g_{a \gamma \gamma}^{4}$. By contrast, an experiment such as CAST will scale only as $\mathcal{N}_{a} g_{\text {arr }}^{4}$ whenever the ALP masses are sufficiently large such that the wave packets corresponding to ALPs of different masses start to separate at the location of the detector, such that the amplitudes no longer add coherently. A dedicated analysis of this effect is beyond the scope of this Letter but the formalism is broadly similar to that of neutrino oscillations.

ALP-induced cavity source terms.- Equation (1) implies that Maxwell's equations are modified in the presence of nonzero $g_{a \gamma \gamma}$ [45]. Ignoring the EH terms for now, the modified equations of motion for $\mathcal{N}_{a}$ ALPs with zero external charges or currents are

$$
\begin{gathered}
\nabla \cdot \mathbf{E}=\mathbf{B} \cdot \sum_{i=1}^{\mathcal{N}_{a}} g_{a \gamma \gamma}^{(i)} \nabla a_{i}, \\
\nabla \times \mathbf{B}=\frac{\partial \mathbf{E}}{\partial t}-\mathbf{E} \times \sum_{i=1}^{\mathcal{N}_{a}} g_{a \gamma \gamma}^{(i)} \nabla a_{i}+\mathbf{B} \sum_{i=1}^{\mathcal{N}_{a}} g_{a \gamma \gamma}^{(i)} \frac{\partial a_{i}}{\partial t}, \\
\left(\partial_{t}^{2}-\nabla^{2}+m_{a_{i}}^{2}\right) a_{i}=g_{a \gamma \gamma}^{(i)} \mathbf{E} \cdot \mathbf{B}\left(i=1, \ldots, \mathcal{N}_{a}\right) .
\end{gathered}
$$

We will assume that the $g_{a \gamma \gamma}^{(i)}$ are small and use classical field perturbation theory. Equation (7) shows that regions of 
nonzero $\mathbf{E} \cdot \mathbf{B}$ will source the $a_{i}$ fields proportional to $g_{a \gamma \gamma}^{(i)}$; Eqs. (5) and (6) imply that $a_{i}$ will in turn source signal fields cubic in the cavity fields and proportional to $\left(g_{a \gamma \gamma}^{(i)}\right)^{2}$. If all the $g_{a \gamma \gamma}^{(i)}$ are identical, the signal fields will be

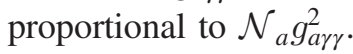

Unless otherwise specified, we now restrict to the case of a single ALP, $\mathcal{N}_{a}=1$. We may use the Green's function for the ALP field to write the signal fields solely in terms of the background fields. The appropriate Green's function is the classical retarded Green's function for the Klein-Gordon equation $G_{R}\left(\mathbf{x}, t, \mathbf{x}^{\prime}, t^{\prime}\right)$. The solution for $a(\mathbf{x}, t)$ is then

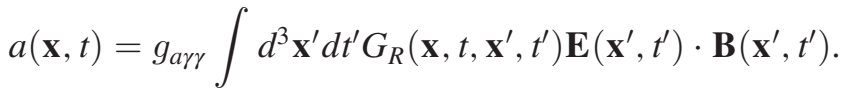

Suppose the cavity is pumped at resonant frequencies $\omega_{1}$ and $\omega_{2}$, with associated modes $\mathbf{E}_{1}, \mathbf{B}_{1}$ and $\mathbf{E}_{2}, \mathbf{B}_{2}$, (Fig. 1). The total pump field is $\mathbf{E}_{\mathrm{p}}=\mathbf{E}_{1} e^{i \omega_{1} t}+\mathbf{E}_{2} e^{i \omega_{2} t}$, where it is understood that the physical field is the real part of the complex field and that the correct phase relationships exist between $\mathbf{E}$ and $\mathbf{B}$. From now on, we will drop the explicit time dependence of the pump modes.

The ALP-dependent terms on the right-hand side of Eqs. (5) and (6) can be interpreted as an effective ALP charge and current:

$\rho_{a}=g_{a \gamma \gamma} \mathbf{B}_{\mathrm{p}} \cdot \nabla a ; \quad \mathbf{J}_{a}=g_{a \gamma \gamma}\left(\nabla a \times \mathbf{E}_{\mathrm{p}}+\mathbf{B}_{\mathrm{p}} \frac{\partial a}{\partial t}\right)$.

Note that since $a$ is quadratic in the pump fields, $\rho_{a}$ and $\mathbf{J}_{a}$ are cubic, with frequency components $\omega_{1}, \omega_{2}, 2 \omega_{1} \pm \omega_{2}$, and $2 \omega_{2} \pm \omega_{1}$. If $\mathbf{E}_{\mathrm{p}}$ and $\mathbf{B}_{\mathrm{p}}$ satisfy Maxwell's equations, then $\rho_{a}$ and $\mathbf{J}_{a}$ satisfy the continuity equation $\partial \rho_{a} / \partial t+$ $\nabla \cdot \mathbf{J}_{a}=0$. Thus, using the solution for $a$ in Eq. (8) with $\mathbf{E}=\mathbf{E}_{\mathrm{p}}$, we may treat $\mathbf{J}_{a}$ as a source for the cavity involving only the pump fields $\mathbf{E}_{\mathrm{p}}$ and $\mathbf{B}_{\mathrm{p}}$, identical in formalism to a real current source involving moving charges.

Signal strength. - To solve for the signal fields, we will use the general formalism of cavity Green's functions [46]. We assume that a signal mode $\omega_{s}$ is a resonant mode of the cavity which matches one of the frequency components of $\mathbf{J}_{a}$, which we take to be $2 \omega_{1}-\omega_{2}$ for concreteness. Assuming a finite quality factor $Q_{s}$ for this mode, the ALPsourced $\mathbf{E}_{a}$ field which develops in a cavity of volume $V$ is

$$
\mathbf{E}_{a}(\mathbf{x})=\frac{Q_{s}}{\omega_{s} V} \hat{\mathbf{E}}_{s}(\mathbf{x}) \int d^{3} \mathbf{x}^{\prime} \hat{\mathbf{E}}_{s}\left(\mathbf{x}^{\prime}\right) \cdot \mathbf{J}_{a}\left(\mathbf{x}^{\prime}\right),
$$

where $\hat{\mathbf{E}}_{s}$ is dimensionless with normalization $\int d^{3} \mathbf{x}\left|\hat{\mathbf{E}}_{s}(\mathbf{x})\right|^{2}=V$.

To estimate the size of the signal, we normalize the pump modes such that $\int d^{3} \mathbf{x}\left|\mathbf{E}_{1}(\mathbf{x})\right|^{2}=\int d^{3} \mathbf{x}\left|\mathbf{E}_{2}(\mathbf{x})\right|^{2}=E_{0}^{2} V$, and write $\mathbf{J}_{a}=\kappa_{m_{a}} E_{0}^{3} \hat{\mathbf{J}}_{a}$, where $\hat{\mathbf{J}}_{a}$ is dimensionless and $\kappa_{m_{a}}$ has dimension -3 . In the two limiting cases of $m_{a} \gg \omega_{s}$ and $m_{a} \ll \omega_{s}$, we choose $\kappa_{m_{a}}$ to be $\kappa_{\infty}=g_{a \gamma \gamma}^{2} \omega_{s} / m_{a}^{2}$ and

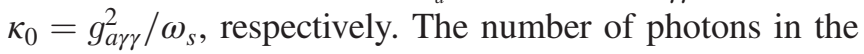
signal field is

$$
N_{s}=\frac{1}{2 \omega_{s}} \int d^{3} \mathbf{x}\left|\mathbf{E}_{a}(\mathbf{x})\right|^{2}=\frac{Q_{s}^{2} V E_{0}^{6}}{2 \omega_{s}^{3}} \kappa_{m_{a}}^{2} K_{m_{a}}^{2},
$$

where we have defined the dimensionless cavity form factor

$$
K_{m_{a}} \equiv \frac{1}{V}\left|\int d^{3} \mathbf{x}^{\prime} \hat{\mathbf{E}}_{s}\left(\mathbf{x}^{\prime}\right) \cdot \hat{\mathbf{J}}_{a}\left(\mathbf{x}^{\prime}\right)\right|
$$

Note that $K$ and $\kappa$ both depend on $m_{a}$ through the Green's function $G_{R}$.

Cavity form factors: Heavy and light ALPs.-To understand the signal strength as a function of $m_{a}$, we compute the cavity form factors in two limits: $K_{\infty}$, where $m_{a} \gg \omega_{s}$, and $K_{0}$, where $m_{a} \ll \omega_{s}$.

In the limit $m_{a} \gg \omega_{s}$, we have $\partial_{t}^{2} a, \nabla^{2} a \ll m_{a}^{2} a$, so we can "integrate out" the ALP by solving algebraically for $a$ in terms of the pump fields. This gives

$\mathbf{J}_{\infty}=\frac{g_{a \gamma \gamma}^{2}}{m_{a}^{2}}\left(\nabla\left(\mathbf{E}_{\mathrm{p}} \cdot \mathbf{B}_{\mathrm{p}}\right) \times \mathbf{E}_{\mathrm{p}}+\mathbf{B}_{\mathrm{p}} \frac{\partial}{\partial t}\left(\mathbf{E}_{\mathrm{p}} \cdot \mathbf{B}_{\mathrm{p}}\right)\right)$.

In the limit $m_{a} \rightarrow 0$, the ALP Green's function is identical to the retarded Green's function familiar from electromagnetism:

$$
a_{0}(\mathbf{x}, t)=\frac{g_{a \gamma \gamma}}{4 \pi} \int \frac{d^{3} \mathbf{x}^{\prime}}{\left|\mathbf{x}-\mathbf{x}^{\prime}\right|} \mathbf{E}_{\mathrm{p}}\left(\mathbf{x}^{\prime}, t_{R}\right) \cdot \mathbf{B}_{\mathrm{p}}\left(\mathbf{x}^{\prime}, t_{R}\right),
$$

where $t_{R}=t-\left|\mathbf{x}-\mathbf{x}^{\prime}\right|$ is the retarded time. In this case, $a$ responds nonlocally to changes in $\mathbf{E}_{\mathrm{p}}$ and $\mathbf{B}_{\mathrm{p}}$, with a time delay given by $t_{R}$. Since the ALP-mediated current $\mathbf{J}_{0}$, which may be computed from Eq. (14) using Eq. (9), is also nonlocal, there is no simple expression in terms of the pump fields.

As an example, consider a right cylindrical cavity with $\omega_{1}=\mathrm{TE}_{011}, \omega_{2}=\mathrm{TM}_{010}$, and $\omega_{s}=2 \omega_{1}-\omega_{2}=\mathrm{TM}_{020}$ (with mode labeling conventions following [46]), satisfied for a cavity of radius $a$ and height $d=3.112 a$. We find $K_{\infty}=0.18$ and $K_{0}=0.24$ (see the Supplemental Material [47] for details), where the latter result assumes that both $\sin \left(\omega_{s} t\right)$ and $\cos \left(\omega_{s} t\right)$ components of the signal can be added in quadrature, appropriate for photon counting at the standard quantum limit. This example demonstrates that the cavity form factor can be relatively insensitive to $m_{a}$, and thus a single cavity can be used to probe a broad range of ALP masses. This is a strong advantage over traditional resonant searches for ALP dark matter [19,20,53,54], which require careful tuning to match a resonance (e.g., a cavity mode or a Larmor frequency) to $m_{a}$. By contrast, once $\omega_{s}=2 \omega_{1}-\omega_{2}$ is accomplished by tuning the cavity geometry, no further tuning is required to set limits on $g_{\text {ary }}$ for any $m_{a}$. 
Expected sensitivity to ALPs.-To estimate the sensitivities in the light and heavy mass limits, we compute the expected number of signal photons $N_{s}$. From Eq. (11) we have

$N_{s, 0}=\frac{Q_{s}^{2} V E_{0}^{6}}{2 \omega_{s}} \frac{g_{a \gamma \gamma}^{4}}{\omega_{s}^{4}} K_{0}^{2}, \quad N_{s, \infty}=\frac{Q_{s}^{2} V E_{0}^{6}}{2 \omega_{s}} \frac{g_{a \gamma \gamma}^{4}}{m_{a}^{4}} K_{\infty}^{2}$.

To measure the signal, we imagine a filtering geometry as suggested in Ref. [44] (and shown in Fig. 1), where at some point in the geometry the pump fields are exponentially suppressed compared to the signal field, which is possible as long as $\omega_{s}>\omega_{1,2}$. At this location, the signal can be measured without contamination from the pump modes (though we note that this is more of a practical concern than an irreducible background, since the pump modes are at a different frequency than the signal). We estimate the signal-to-noise ratio (SNR) with the Dicke radiometer equation, neglecting any information about the field phase:

$$
\mathrm{SNR}=\frac{P_{s}}{T} \sqrt{\frac{t}{B}} \approx \frac{N_{s}}{N_{\mathrm{th}}} \frac{1}{2 L Q_{s}} \sqrt{\frac{t}{B}},
$$

where $P_{s}$ is the signal power, $t$ is the total measurement time, $B$ is the signal bandwidth, $L$ is the length of the cavity, and $N_{\text {th }}=T / \omega_{s}$ is the number of thermal photons at the signal frequency (valid for temperatures $T \gg \omega_{s}$, and assuming thermal noise dominates). A detailed sensitivity calculation exploiting our knowledge of the pump field phases, perhaps using phase-sensitive amplifiers instead of photon counting, will be presented in a future work.

Our expected sensitivity to $g_{a \gamma \gamma}$ is then

$g_{a \gamma \gamma}^{\lim _{a \gamma}}=\left(\frac{4 T L}{Q_{s} V E_{0}^{6}} \sqrt{\frac{B}{t}} \mathrm{SNR}\right)^{1 / 4} \times \begin{cases}K_{0}^{-1 / 2} \omega_{s}, & m_{a} \ll \omega_{s} \\ K_{\infty}^{-1 / 2} m_{a}, & m_{a} \gg \omega_{s} .\end{cases}$

In an actual experimental implementation, a cavity should be designed specifically to maximize the figure of merit in Eq. (17) while minimizing issues such as multipacting, dark currents, field emission, intermodulation in the feed lines, and surface nonlinearities $[55,56]$.

For a fixed choice of modes and cavity size (hence fixed $\omega_{s}$ and $V$ ), the reach is constant at small $m_{a}$ and degrades linearly at large $m_{a}$. There is, in principle, some dependence of $K_{m_{a}}$ on $m_{a}$, but with a suitable choice of modes this dependence is extremely mild. For a large number $\mathcal{N}_{a}$ of light ALPs, all with $m_{a} \ll \omega_{s}$, Eq. (17) should be inter-

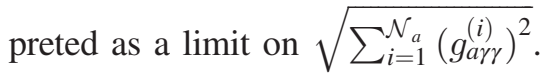

Phase 1: Conservative projected reach.-We envision our experiment progressing in three stages, each building on current technology. For phase 1, we take the following parameters: cavity temperature $T=1.5 \mathrm{~K}$; a right cylindrical cavity with $a=0.5 \mathrm{~m}$ and the $\mathrm{TE}_{011} / \mathrm{TM}_{010} / \mathrm{TM}_{020}$ mode combination, giving $d=1.56 \mathrm{~m}, f_{s}=\omega_{s} /(2 \pi)=527 \mathrm{MHz}$ and $V=1.23 \mathrm{~m}^{3} ; K_{0}=0.24$ as calculated above; pump field strength $E_{0}=45 \mathrm{MV} / \mathrm{m}$; and a cavity bandwidth of $f_{s} / Q_{s}=2 \mathrm{~Hz}$, corresponding to $Q_{s}=2.6 \times 10^{8}$. This $Q$ is much smaller than what typical high-performance SRF cavities can achieve, but a wide cavity response function for phase 1 allows the frequency-matching condition $\omega_{s}=$ $2 \omega_{1}-\omega_{2}$ to be approximately satisfied even if vibrational distortions shift $\omega_{s}$ by $\mathcal{O}(\mathrm{Hz})$. Note also that our mode combination satisfies $\omega_{s}>\omega_{1}, \omega_{2}$, making it amenable to filtering; to model this, we assume the total cavity length is twice the cavity height, $L=3.11 \mathrm{~m}$.

At $1.5 \mathrm{~K}$, the thermal noise in the signal mode is $N_{\text {th }}=$ $k T / \omega_{s} \simeq 60$ photons. A lower operating temperature would be desirable, but the cooling power requirements are substantial: assuming that the pump modes have $Q_{1,2}=10^{12}$, characteristic of the best $Q$ achieved in SRF cavities [57,58], the cavity lifetime for the pump modes is $\tau_{1,2}=$ $Q_{1,2} / f_{1,2} \sim 2600 \mathrm{~s}$ and the power dissipated is $\mathcal{O}(10 \mathrm{~W})$. Dilution refrigerators, which have a cooling capacity of $\mathcal{O}(\mathrm{mW})$, are not sufficient, and the cavity must operate at liquid helium temperatures.

We first consider the case where the injected pump bandwidth is comparable to the cavity bandwidth, $B=2 \mathrm{~Hz}$. For light ALPs, the OSQAR bound can be surpassed by nearly an order of magnitude in a measurement of a single cavity lifetime of $\tau_{s}=Q_{s} / f_{s}=0.5 \mathrm{~s}$. Integrating the signal over a time $t \sim 1$ day, we can obtain a phase 1 reach of $g_{a \gamma \gamma, 0}^{\lim }=1.2 \times 10^{-9} \mathrm{GeV}^{-1}$. For $m_{a} \gg \omega_{s}$, we can get the limits by using $g_{a \gamma \gamma, \infty}^{\lim }=\left(m_{a} / \omega_{s}\right) \sqrt{K_{0} / K_{\infty}} g_{a \gamma \gamma, 0}^{\lim }$.

One could also pump the cavity with a bandwidth narrower than the cavity itself, for example, by locking the pump tones to an atomic clock. Taking $B=1 / t$, the narrowest allowed bandwidth for a given measurement time $t$, a bound of $g_{a \gamma \gamma, 0}^{\lim }=2.6 \times 10^{-10} \mathrm{GeV}^{-1}$ could be reached in a day. In the case $B=1 / t$, the SNR scales linearly with time, so the limit on $g_{\text {ary }}$ scales as $t^{1 / 4}$. The two bandwidth choices for phase 1 are shown in Fig. 2; we have not explicitly calculated the reach for $m_{a} \sim \omega_{s}$ (shown as dashed lines), but we expect the light and heavy mass limits to be excellent approximations away from this region.

Phase 2: Detecting the Euler-Heisenberg contribution.-As we have discussed, there is an irreducible contribution to cubic nonlinearities in Maxwell's equations from the EH Lagrangian, see Eq. (3). The effective EH charge and current are $[43,59]$

$$
\rho_{\mathrm{EH}}=-\frac{4 \alpha^{2}}{45 m_{e}^{4}} \nabla \cdot \mathbf{P} ; \quad \mathbf{J}_{\mathrm{EH}}=\frac{4 \alpha^{2}}{45 m_{e}^{4}}\left(\nabla \times \mathbf{M}+\frac{\partial \mathbf{P}}{d t}\right),
$$

with $\mathbf{P}=7(\mathbf{E} \cdot \mathbf{B}) \mathbf{B}+2\left(\mathbf{E}^{2}-\mathbf{B}^{2}\right) \mathbf{E}$ and $\mathbf{M}=7(\mathbf{E} \cdot \mathbf{B}) \mathbf{E}-$ $2\left(\mathbf{E}^{2}-\mathbf{B}^{2}\right) \mathbf{B}$. The number of photons from the EH signal can be estimated similarly to the ALP case.

For phase 2, we assume the cylindrical cavity geometry from phase 1, but with $Q_{s}=10^{12}$. Indeed, in tuned SQUID 


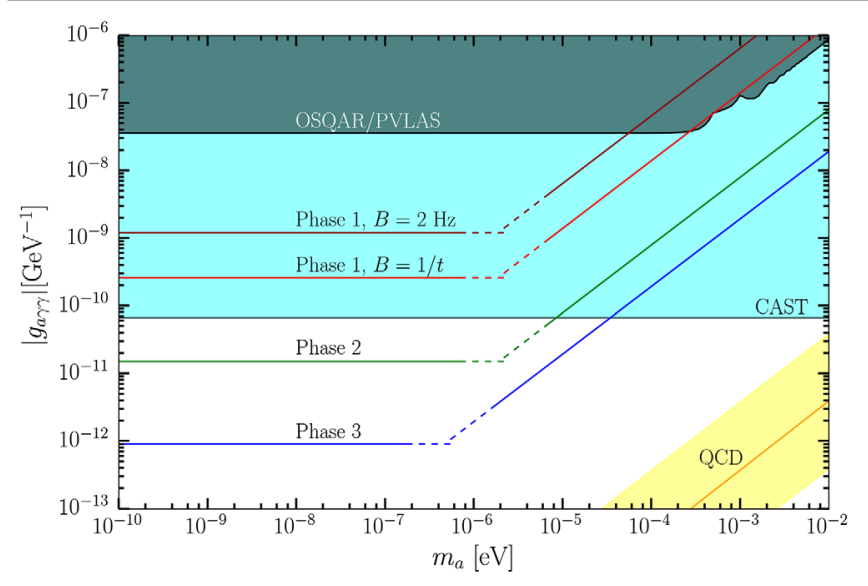

FIG. 2. Projected sensitivities of our proposal (approximations for $m_{a} \sim \omega_{s}$ shown in dashed lines), along with existing constraints [36-38]. See text for details.

magnetometers, a feedback circuit may be used to broaden the bandwidth without sacrificing $Q$ [60]; such a scheme may be possible here. We find that

$$
N_{\mathrm{EH}}=\frac{Q_{s}^{2} V E_{0}^{6}}{2 \omega_{s}^{3}} \kappa_{\mathrm{EH}}^{2} K_{\mathrm{EH}}^{2} \approx 3.6,
$$

with $\kappa_{\mathrm{EH}}=4 \alpha^{2} \omega_{s} /\left(45 m_{e}^{4}\right)$ and $K_{\mathrm{EH}}=(1 / V) \int d^{3} \mathbf{x}^{\prime} \hat{\mathbf{J}}_{\mathrm{EH}}$. $\hat{\mathbf{E}}_{s}=0.18$, with $\hat{\mathbf{J}}_{\mathrm{EH}}$ defined analogously to $\hat{\mathbf{J}}_{a}$. This signal strength is roughly consistent with Ref. [44] given our different choices of parameters and modes. Therefore, assuming $B=1 / t$, the EH signal can be detected within 20 days of running. The corresponding sensitivity to light ALPs for the same integration time is $g_{a \gamma \gamma, 0}^{\lim }=1.6 \times$ $10^{-11} \mathrm{GeV}^{-1}$; this is shown in Fig. 2. This would surpass the CAST bound of $g_{\text {ary }}=6.6 \times 10^{-11} \mathrm{GeV}^{-1}$ and would also be competitive with recent proposals to search for ALP DM at low masses such as ABRACADABRA [21,61]. In some models, an ALP with these couplings could also be the QCD axion $[62,63]$. If a positive signal were detected, the ALP nature of the signal could be verified using a second cavity with different mode combinations. If the ALP is heavier than $\omega_{s}$, the combination of the two measurements would suffice to determine both $g_{a \gamma \gamma}$ and $m_{a}$.

Naively, the sensitivity of this proposal to probe ALPs becomes limited when $N_{\mathrm{EH}} \sim N_{s}$. In principle, one can search for the ALP signal on the top of the thermal and EH backgrounds, but as with the "neutrino floor" in WIMP direct detection experiments, the SNR will grow much slower than $t^{1 / 4}$. However, with a slightly different mode choice, the EH contribution can be removed, leaving behind only the ALP signal. The idea is to pump an additional mode degenerate with $\omega_{1}$ but with a different field configuration. By tuning the three different pump amplitudes, we can arrange to have $K_{\mathrm{EH}}=0$ with $K_{m_{a}} \neq 0$. For these special pump amplitudes, the EH contribution to light-by-light scattering vanishes at amplitude level, and there is no interference with the ALP amplitude. We give a proof of principle demonstration of this idea in the Supplemental Material [47].

Phase 3: Probing the axiverse.-As the optimistic endpoint of this proposed program of experiments, consider a large cylindrical cavity with $a=2$ and $d=6.22 \mathrm{~m}$, giving $f_{s}=132 \mathrm{MHz}$, with the same mode combinations as considered in phases 1 and 2 and $Q_{s}=10^{12}$. We suppose a cavity geometry can be developed which permits $K_{0} \sim$ 0.24 with the EH contribution tuned away to sufficient precision as described in phase 2 , and a compact filtering geometry with length $L=10 \mathrm{~m}$. Assuming the same pump strength as phases 1 and 2 , and integrating for a total time $t=1$ year with $B=1 / t$, we find from Eq. (17) a maximum sensitivity at low masses of $g_{a \gamma \gamma, 0}^{\lim } \sim 9.1 \times 10^{-13} \mathrm{GeV}^{-1}$, shown in Fig. 2.

Revisiting the axiverse scenario, suppose that $\mathcal{N}_{a}$ ALPs all had decay constants $f_{a}$ at the string scale, which we conservatively take to be the renormalized Planck scale, $10^{18} \mathrm{GeV} / \sqrt{\mathcal{N}_{a}}$. These string ALPs would have photon couplings of $g_{\text {ary }}=\alpha / f_{a} \sim \sqrt{\mathcal{N}_{a}} 10^{-20} \mathrm{GeV}^{-1}$. Our phase 3 would be sensitive to $g_{a \gamma \gamma} \sqrt{\mathcal{N}_{a}} \sim 10^{-20} \mathrm{GeV}^{-1} \times \mathcal{N}_{a}$, which could bound the number of string-scale ALPs with masses less than $10^{-6} \mathrm{eV}$ by $\mathcal{N}_{a} \lesssim 10^{8}$. While this is still (much) larger than typical expectations from string theory, one could still imagine placing constraints on particular compactification geometries which contain large numbers of nontrivial cycles [64], allowing low-energy SRF cavity experiments to offer a fascinating probe into the ultra-highenergy regime of quantum gravity and the landscape of string theory vacua.

A. H., Y. K., and Y.S. thank Ben Safdi and the University of Michigan Slack channel for facilitating discussion in the early stages of this work. Y. K. thanks Prateek Agrawal, M. C. David Marsh, and the participants of the workshop "Axions in Stockholm-Reloaded" for discussions about the axiverse, and James Halverson and Cody Long for discussions about axions in string compactifications. We thank Daniel Bowring, Fritz Caspers, Aaron Chou, Anna Grassellino, Roni Harnik, Kent Irwin, Akira Miyazaki, Jonathan Ouellet, Sam Posen, Alexander Romanenko, and Slava Yakovlev for enlightening discussions regarding cavity design and photon readout. We thank Junwu Huang, Gilad Perez and Jesse Thaler for helpful comments, and Lindley Winslow for support in the early stages of this project. A. H. is supported in part by the NSF under Grant No. PHY-1620074 and by the Maryland Center for Fundamental Physics (MCFP). The work of Z. B. is supported by the National Science Foundation under Grant No. NSF PHY-1806440. This work was supported in part by the Kavli Institute for Cosmological Physics at the University of Chicago through an endowment from the Kavli Foundation and its founder Fred Kavli. 
*zbogorad@mit.edu

thook@umd.edu

¥ykahn@uchicago.edu

§yotam.soreq@cern.ch

[1] R. D. Peccei and H. R. Quinn, Phys. Rev. Lett. 38, 1440 (1977).

[2] S. Weinberg, Phys. Rev. Lett. 40, 223 (1978).

[3] F. Wilczek, Phys. Rev. Lett. 40, 279 (1978).

[4] P. Sikivie, Lect. Notes Phys. 741, 19 (2008).

[5] J. E. Kim and G. Carosi, Rev. Mod. Phys. 82, 557 (2010).

[6] A. Hook, arXiv:1812.02669.

[7] P. Svrcek and E. Witten, J. High Energy Phys. 06 (2006) 051.

[8] A. Arvanitaki, S. Dimopoulos, S. Dubovsky, N. Kaloper, and J. March-Russell, Phys. Rev. D 81, 123530 (2010).

[9] J. Preskill, M. B. Wise, and F. Wilczek, Phys. Lett. 120B, 127 (1983).

[10] L. F. Abbott and P. Sikivie, Phys. Lett. 120B, 133 (1983).

[11] M. Dine and W. Fischler, Phys. Lett. 120B, 137 (1983).

[12] P. W. Graham, D. E. Kaplan, and S. Rajendran, Phys. Rev. Lett. 115, 221801 (2015).

[13] R. S. Gupta, Z. Komargodski, G. Perez, and L. Ubaldi, J. High Energy Phys. 02 (2016) 166.

[14] A. Hook and G. Marques-Tavares, J. High Energy Phys. 12 (2016) 101.

[15] O. Davidi, R. S. Gupta, G. Perez, D. Redigolo, and A. Shalit, J. High Energy Phys. 08 (2018) 153.

[16] A. Banerjee, H. Kim, and G. Perez, arXiv:1810.01889.

[17] P. W. Graham, I. G. Irastorza, S. K. Lamoreaux, A. Lindner, and K. A. van Bibber, Annu. Rev. Nucl. Part. Sci. 65, 485 (2015).

[18] I. G. Irastorza and J. Redondo, Prog. Part. Nucl. Phys. 102, 89 (2018).

[19] L. Zhong et al. (HAYSTAC Collaboration), Phys. Rev. D 97, 092001 (2018).

[20] N. Du et al. (ADMX Collaboration), Phys. Rev. Lett. 120, 151301 (2018).

[21] J. L. Ouellet et al., Phys. Rev. Lett. 122, 121802 (2019).

[22] W. DeRocco and A. Hook, Phys. Rev. D 98, 035021 (2018).

[23] H. Liu, B. D. Elwood, M. Evans, and J. Thaler, arXiv: 1809.01656

[24] M. S. Pshirkov, J. Exp. Theor. Phys. 108, 384 (2009).

[25] A. Hook, Y. Kahn, B. R. Safdi, and Z. Sun, Phys. Rev. Lett. 121, 241102 (2018).

[26] F. P. Huang, K. Kadota, T. Sekiguchi, and H. Tashiro, Phys. Rev. D 97, 123001 (2018).

[27] B. R. Safdi, Z. Sun, and A. Y. Chen, arXiv:1811.01020 [Phys. Rev. D (to be published)].

[28] A. Caputo, C. P. Garay, and S. J. Witte, Phys. Rev. D 98, 083024 (2018).

[29] A. Caputo, M. Regis, M. Taoso, and S. J. Witte, J. Cosmol. Astropart. Phys. 03 (2019) 027.

[30] S. Evans and J. Rafelski, Phys. Lett. B 791, 331 (2019).

[31] W. Heisenberg and H. Euler, Z. Phys. 98, 714 (1936).

[32] J. S. Schwinger, Phys. Rev. 82, 664 (1951).

[33] D. L. Burke et al., Phys. Rev. Lett. 79, 1626 (1997).

[34] M. Aaboud et al. (ATLAS Collaboration), Nat. Phys. 13, 852 (2017).
[35] D. Bernard, Nuovo Cimento A 110, 1339 (1997).

[36] R. Ballou et al. (OSQAR Collaboration), Phys. Rev. D 92, 092002 (2015).

[37] F. Della Valle, A. Ejlli, U. Gastaldi, G. Messineo, E. Milotti, R. Pengo, G. Ruoso, and G. Zavattini, Eur. Phys. J. C 76, 24 (2016).

[38] V. Anastassopoulos et al. (CAST Collaboration), Nat. Phys. 13, 584 (2017).

[39] J. W. Brockway, E. D. Carlson, and G. G. Raffelt, Phys. Lett. B 383, 439 (1996).

[40] J. A. Grifols, E. Masso, and R. Toldra, Phys. Rev. Lett. 77, 2372 (1996).

[41] A. Payez, C. Evoli, T. Fischer, M. Giannotti, A. Mirizzi, and A. Ringwald, J. Cosmol. Astropart. Phys. 02 (2015) 006.

[42] M. C. D. Marsh, H. R. Russell, A. C. Fabian, B.P. McNamara, P. Nulsen, and C.S. Reynolds, J. Cosmol. Astropart. Phys. 12 (2017) 036.

[43] G. Brodin, M. Marklund, and L. Stenflo, Phys. Rev. Lett. 87, 171801 (2001).

[44] D. Eriksson, G. Brodin, M. Marklund, and L. Stenflo, Phys. Rev. A 70, 013808 (2004).

[45] P. Sikivie, Phys. Rev. Lett. 51, 1415 (1983); 52, 695(E) (1984).

[46] D. Hill, Electromagnetic Fields in Cavities: Deterministic and Statistical Theories, IEEE Press Series on Electromagnetic Wave Theory (Wiley Collaboration, Hoboken, New Jersey, USA, 2009).

[47] See Supplemental Material at http://link.aps.org/ supplemental/10.1103/PhysRevLett.123.021801 for further details about the example mode choices we have used to calculate the cavity form factors, as well as a comparison of our proposal to LSW experiments as well as multimode pumping for axion DM (Refs. [48-52]).

[48] R. Harnik, SRF-Based Dark Matter Search: Theory Motivation, URL https://indico.fnal.gov/event/19433/session/2/ contribution $/ 1 /$ material/slides/0.pdf.

[49] A. Grassellino, SRF-Based Dark Matter Search: Experiment, URL https://indico.fnal.gov/event/19433/session/2/ contribution/2/material/slides/0.pdf.

[50] P. Sikivie, arXiv:1009.0762.

[51] M. Goryachev, B. Mcallister, and M.E. Tobar, arXiv: 1806.07141 .

[52] M. Goryachev, B. Mcallister, and M. E. Tobar, Phys. Dark Universe 23, 100244 (2019).

[53] P. Sikivie, N. Sullivan, and D. B. Tanner, Phys. Rev. Lett. 112, 131301 (2014).

[54] D. F. Jackson Kimball et al., arXiv:1711.08999.

[55] H. Padamsee, Supercond. Sci. Technol. 14, R28 (2001).

[56] H. Padamsee, RF Superconductivity: Science, Technology, and Applications (John Wiley \& Sons, Weinheim, Germany, 2009).

[57] A. Romanenko, A. Grassellino, A. C. Crawford, D. A. Sergatskov, and O. Melnychuk, Appl. Phys. Lett. 105, 234103 (2014).

[58] A. Romanenko, R. Pilipenko, S. Zorzetti, D. Frolov, M. Awida, S. Posen, and A. Grassellino, arXiv: 1810.03703.

[59] M. Soljacic and M. Segev, Phys. Rev. A 62, 043817 (2000). 
[60] W. Myers, D. Slichter, M. Hatridge, S. Busch, M. Mößle, R. McDermott, A. Trabesinger, and J. Clarke, J. Magn. Reson. 186, 182 (2007).

[61] Y. Kahn, B. R. Safdi, and J. Thaler, Phys. Rev. Lett. 117, 141801 (2016).
[62] M. Farina, D. Pappadopulo, F. Rompineve, and A. Tesi, J. High Energy Phys. 01 (2017) 095.

[63] P. Agrawal, J. Fan, M. Reece, and L.-T. Wang, J. High Energy Phys. 02 (2018) 006.

[64] M. R. Douglas and S. Kachru, Rev. Mod. Phys. 79, 733 (2007). 